\title{
Racial and Ethnic Disparities in Oncotype DX Test Receipt in a Statewide Population-Based Study
}

\author{
Brigette A. Davis, MPH; ; Jenerius A. Aminawung, MD, MPH ${ }^{\mathrm{a}, \mathrm{b}}$; Maysa M. Abu-Khalaf, MD, MBBS ${ }^{\mathrm{a}, \mathrm{c}}$; \\ Suzanne B. Evans, MD, MPH ${ }^{\mathrm{a}, \mathrm{c}}$; Kevin Su, MDª Rajni Mehta, $\mathrm{MPH}^{\mathrm{d}}$; Shi-Yi Wang, MD, PhD a,e \\ and Cary P. Gross, MDa,b
}

\begin{abstract}
Background: Racial disparities have been reported in breast cancer care, yet little is known about disparities in access to gene expression profiling (GEP) tests. Given the impact of GEP test results, such as those of Oncotype DX (ODx), on treatment decision-making for hormone receptor-positive $(\mathrm{HR}+)$ breast cancer, it is particularly important to assess disparities in its use. Methods: We conducted a retrospective population-based study of 8,784 patients diagnosed with breast cancer in Connecticut during 2011 through 2013 . We assessed the association between race, ethnicity, and ODx receipt among women with HR+ breast cancer for whom NCCN does and does not recommend ODx testing, using bivariate and multivariate logistic analyses. Results: We identified 5,294 women who met study inclusion criteria: $83.8 \%$ were white, $6.3 \%$ black, and $7.4 \%$ Hispanic. Overall, $50.9 \%(n=4,131)$ of women in the guideline-recommended group received ODx testing compared with $18.5 \%(n=1,163)$ in the nonrecommended group. More white women received the ODx test compared with black and Hispanic women in the recommended and nonrecommended groups $(51.4 \%$ vs $44.6 \%$ and $47.7 \%$; and $21.2 \%$ vs $9.0 \%$ and $9.7 \%$, respectively). After adjusting for tumor and clinical characteristics, we observed significantly lower ODx use among black (odds ratio [OR], 0.64; $95 \% \mathrm{Cl}, 0.47-0.88)$ and Hispanic women $(\mathrm{OR}, 0.59 ; 95 \% \mathrm{Cl}, 0.45-0.77)$ compared with white women in the recommended group and in the guideline-discordant group (blacks: OR, $0.39 ; 95 \% \mathrm{Cl}, 0.20-0.78$, and Hispanics: OR, $0.44 ; 95 \% \mathrm{Cl}, 0.23-0.85$ ). Conclusions: In this populationbased study, we identified racial disparities in ODx testing. Disparities in access to innovative cancer care technologies may further exacerbate existing disparities in breast cancer outcomes.
\end{abstract}

J Nat/ Compr Canc Netw 2017;15(3):346-354

\section{Background}

Racial disparities are pervasive in medical care and are particularly well documented throughout the trajectory of breast cancer diagnosis and treatment. ${ }^{1,2}$ Disparities in screening, follow-up, rates of referral, receipt of surgery, types of treatment, time to treatment, and aftercare have been reported for racial and ethnic minorities and women of lower socioeconomic status. ${ }^{1,3-7}$ It is likely that disparities are compounded at each stage along

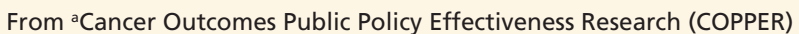
Center, Yale University; 'bection of General Internal Medicine, Yale University; 'Smilow Cancer Hospital, Yale New Haven Hospital; 'Yale Cancer Center Rapid Case Ascertainment Center; and 'Yale School of Public Health, New Haven, Connecticut.

Submitted June 16, 2016; accepted for publication November 8, 2016. Dr. Gross received support from a Research Scholar Grant, RSG-14-02901-CPHPS from the American Cancer Society, and receives support from Medtronic, Inc., Johnson \& Johnson, Pfizer, and Century Oncology. Dr. Abu-Khalaf receives research support from Genentech, Novartis, Merck, Merrimack, and Clovis Oncology; these supports were not used for any portion of the manuscript. The remaining authors have disclosed that the cancer care continuum, and clinical advancements responsible for improved breast cancer outcomes have the potential to exacerbate disparities. ${ }^{8}$ These concerns are supported by evidence suggesting that disparities by race and ethnicity in 5-year relative survival rates are significantly greater in cancer sites more "amenable" to treatment, such as breast and prostate cancer, and almost nonexistent in "low-amenability" sites, such as the pancreas and brain. ${ }^{9}$

they have no financial interests, arrangements, affiliations, or commercial interests with the manufacturers of any products discussed in this article or their competitors.

Author contributions: Project conception and initiation: Gross, Aminawung. Data acquisition: Rajni. Anaylsis: Aminawung. Drafting of manuscript: Davis, Aminawung. Interpretation of data and manuscript revision, review, and approval: Davis, Aminawung, Abu-Khalaf, Evans, $\mathrm{Su}$, Mehta, Wang, Gross. Dr. Gross is the guarantor and affirms that the manuscript is an honest and accurate account of the study being reported. Correspondence: Cary P. Gross, MD, Section of General Internal Medicine, Yale University School of Medicine, P.O. Box 208056, New Haven, CT 06520 E-mail: cary.gross@yale.edu 
Disparities in Oncotype DX Adoption

Substantive advances in the understanding of cancer characteristics that affect prognosis and response to treatment have resulted in risk stratification capabilities that are revolutionizing cancer care, heralding an era of personalized medicine. Gene expression profiling (GEP) tests are one such innovation, and measure the expression of multiple genes simultaneously, unlike single-gene and molecular assays. The most widely used GEP test is the Oncotype DX test (ODx) from Genomic Health (Redwood City, CA). ODx quantifies the expression of 21 genes (16 cancer-related genes and 5 reference genes) to generate a recurrence score (RS). Studies have shown that the RS is both prognostic - it correlates with 10 -year risk of distant recurrence-and predictive of the degree to which adjuvant chemotherapy will reduce the risk of recurrence. ${ }^{10}$

Use of ODx has been incorporated into clinical practice recommendations, such as the NCCN Clinical Practice Guidelines in Oncology (NCCN Guidelines) for Breast Cancer. ${ }^{11}$ Specifically, NCCN recommends considering the use of ODx in patients at a moderate risk for recurrence in whom chemotherapy is likely to be beneficial. The 2009 NCCN Guidelines recommended GEP testing be considered in women with localized hormone receptor-positive $(\mathrm{HR}+)$, HER2-negative (HER2-) invasive breast cancer that is $>5 \mathrm{~mm}$ and either node-negative or node-positive with only micrometastasis ( $\leq 2-\mathrm{mm}$ lymph nodes). ODx was not recommended for patients with HR+ tumors that were particularly low-risk (ie, $\leq 5 \mathrm{~mm}$ ) or were higher-risk (node-positive with $>2 \mathrm{~mm}$ lymph nodes, or macrometastasis), because results would be unlikely to change the course of treatment. ${ }^{12}$ The test was also not recommended for hormone receptornegative (HR-) or metastatic breast cancer. Notably, these recommendations are evolving over time. The NCCN Guidelines were updated in 2015 to include a footnote that allows consideration of GEP in women with 1 to 3 positive axillary nodes. ${ }^{13}$

Given the evolving standards of care regarding GEP use, it is important to understand how it has disseminated into practice, with an emphasis on guidelines that are applicable during the period of dissemination.

Several studies have documented the adoption of ODx, with approximately one-third of eligible patients receiving testing by 2010. ${ }^{14-22}$ However, there are conflicting findings regarding disparities in $\mathrm{ODx}$ use. A recent study of patients at hospitals that were members of the National Cancer Data Base ${ }^{23}$ and a study of trial participants ${ }^{20}$ found racial disparities in ODx testing. Other studies, primarily focused on the early adoption of ODx up to 2010, have also identified disparities based on race, ${ }^{17,19}$ income, ${ }^{14,19}$ and hospital type. ${ }^{17,20}$ In contrast, a recent analysis of the Carolina Breast Cancer Study from 2008 to $2014,{ }^{16}$ a study using SEER-Medicare data from 2005-2009, ${ }^{21}$ and 2 small retrospective studies ${ }^{15,22}$ have identified no racial or socioeconomic disparities in ODx testing. Fewer studies have measured disparities in uptake by patients of Hispanic ethnicity, with some findings showing decreased use $\mathrm{e}^{24}$ and others reporting no difference compared with non-Hispanic whites. ${ }^{25}$ Other studies of access to healthcare have found that Hispanics in the United States experience decreased access to care, similar to African Americans. ${ }^{26,27}$ To address these contradictory findings and gaps in the research, recent population-based data are needed.

In addition to analyzing disparities in access to ODx among patients for whom the test is recommended, it is also important to further our understanding of the "overuse" of ODx. For instance, NCCN Guidelines during the 2011-2013 period did not recommend considering ODx for patients with higher-risk disease, such as macrometastatic-positive nodes. Two studies estimate that 20\% to $39 \%$ of tests are ordered outside of NCCN recommendations, ${ }^{18,21}$ but few studies have assessed sociodemographic determinants of guideline-discordant testing. The Carolina Breast Cancer Study found that black patients with node-positive disease were less likely than their white counterparts to receive testing. ${ }^{16}$ However, it is unclear whether this is due to sample selection and whether similar differences would be seen in a population-based study sample.

This study builds on current research on ODx use and disparities in 2 key ways: (1) identifying disparities in a statewide, population-based analysis, and (2) identifying guideline-discordant use and disparate uptake based on sociodemographic characteristics.

\section{Methods}

\section{Data Source and Study Sample}

We conducted a retrospective population-based analysis using data collected through the Yale Cancer Rapid Case Ascertainment Shared Resource 
Davis et al

(RCA) and its relationship to the Connecticut Tumor Registry (CTR). Under this relationship, following study approval, the hospitals grant the RCA access to medical records for review. The RCA was developed in 1986 in response to the Connecticut Hospital Association's request to establish a single entity that would be responsible for all aspects of population-based cancer epidemiology studies. All hospitals and private pathology laboratories in Connecticut are required by law to report cancer cases to the CTR. We used CTR data located in the Connecticut Department of Public Health on reported cases of incident breast cancer for 2011 through 2013. Hospitals and laboratories each keep a log of patients whose samples have been sent for GEP testing. We abstracted information on ODx testing from GEP requisition and testing logs, and ODx results from medical records, amended pathology reports, GEP testing logs, and Genomic Health (Redwood City, CA) reports at hospitals that reported incident breast cancer cases during the study period.

We identified 8,784 women with newly diagnosed breast cancer from 2011 through 2013. We included women with localized HR + breast cancer for whom the 2009 NCCN Guidelines recommended considering $\mathrm{ODx}$ testing and those for whom the test was not recommended. ${ }^{12}$ Specifically, we included in the guideline-concordant group women with localized estrogen receptor-positive (ER+), HER2- invasive cancer breast that was either node-negative or micrometastatic and $>5 \mathrm{~mm}$, and in the guidelinediscordant group we included women with localized node-positive ( $>2 \mathrm{~mm}$ lymph nodes) invasive breast cancer irrespective of tumor size. Based on the 2009 guidelines, women with 1 to 3 positive axillary nodes were considered guideline-discordant. We excluded patients with ductal carcinoma in situ, localized $\mathrm{HR}+$, node-negative, $\leq 5 \mathrm{~mm}$, localized $\mathrm{HR}-$, and metastatic breast cancer at diagnosis (Figure 1). Patients were also excluded if the cancer was diagnosed through autopsy. Our study was approved by the Yale University and the Connecticut Department of Public Health Human Investigation Committees and hospital Institutional Review Boards.

\section{Construction of Variables}

Patient Characteristics: Patient characteristics in the CTR reports include age, sex, race, ethnicity, marital status, insurance at time of diagnosis, and

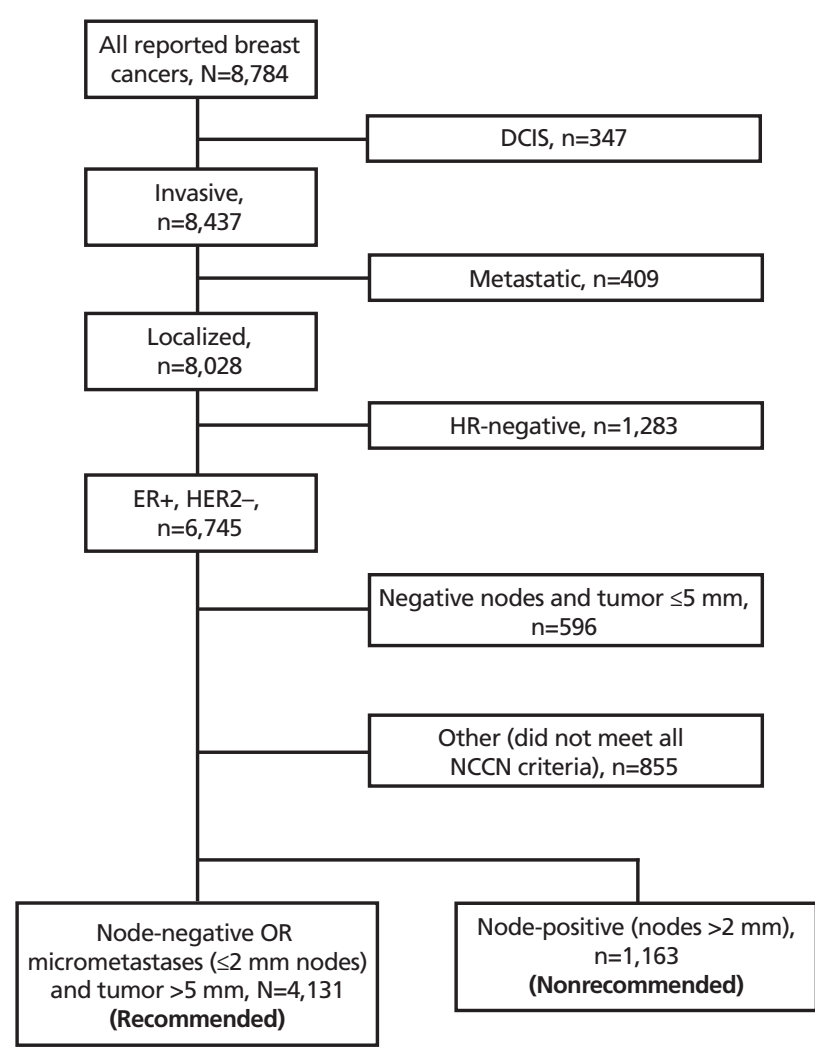

Figure 1. Study sample construction.

Abbreviations: DCIS, ductal carcinoma in situ; ER, estrogen receptor; $H R$, hormone receptor.

socioeconomic status (percent of families living below the poverty level) derived from patient census tract. We categorized poverty into 3 levels $(<5 \%$, $5 \%-10 \%,>10 \%)$ and patient age into 4 age groups $(<50,50-64,65-74, \geq 75$ years $)$. We categorized patient race/ethnicity into Hispanic and non-Hispanic white, black, or other racial groups. Marital status was grouped into married and not married (including single, separated, divorced, widowed, and unmarried partner). Insurance was classified as no insurance, insurance, and other (if insurance status was unknown).

Cancer Characteristics: Tumor characteristics of interest from the CTR included HR status (ER and progesterone receptor), HER2 status, tumor size, histologic type, lymph node size, and histologic grade. HR and HER2 status was dichotomized into positive and not positive (eg, equivocal and negative). We categorized tumor size into $<5 \mathrm{~mm}, 5$ to $9 \mathrm{~mm}, 10$ to $29 \mathrm{~mm}$, and $\geq 30 \mathrm{~mm}$. Using breast cancer characteristics, we identified patients for whom NCCN Guidelines recommended considering ODx use: those with localized 
Disparities in Oncotype DX Adoption

ER + , HER2-, node-negative or nodal micrometastasis, tumors $>5 \mathrm{~mm}$; and patients for whom ODx testing was not recommended: those with localized ER+, node-positive tumors with macrometastasis $(>2 \mathrm{~mm}$ metastatic focus in a lymph node).

Outcome Variable: Our main outcome variable was receipt of ODx abstracted from GEP testing logs, requisition forms, and Genomic Health reports at the reporting hospitals. Patients for whom no ODx testing information could be abstracted from any of the reporting hospitals were considered as not receiving the test.

\section{Analyses}

We used descriptive statistics to characterize receipt of ODx in localized invasive breast cancer for individual patient and tumor factors of interest. We compared receipt of recommended and nonrecommended ODx tests within patient characteristics using $\mathrm{t}$ and chi-square tests as appropriate. We then used logistic regression models to assess the independent association between race and NCCN Guideline-concordant and NCCN Guideline-discordant ODx testing. Race or ethnicity was the key independent variable in our model. Individual patient and tumor characteristics that showed a significant association with ODx use in bivariate analysis were included as covariates in the logistic regression model. We examined the likelihood of receiving the ODx test based on NCCN recommendation group, controlling for age and cancer characteristics (tumor size, grade, and nodal size). Because of the substantial correlation between race and poverty in this study population, we chose to exclude neighborhood poverty to avoid multicollinearity and overcontrolling for race in our final model. ${ }^{28}$ All analyses were performed using SAS version 9.2 (SAS Institute Inc., Cary, NC)

\section{Results}

After restricting the study population based on the NCCN-recommended and NCCN-nonrecommended $\mathrm{HR}+$, node-positive groups, 5,294 women remained in our analysis. The mean age was 63 years (range, 21-106 years), most were white (84\%), and $22 \%$ of the women resided in neighborhoods with $\geq 10 \%$ of families living below the poverty level.
Most of the reported invasive breast cancers (79\%) were $\geq 10 \mathrm{~mm}$ or had no nodal involvement $(73 \%$; Table 1). Among women for whom the ODx test was recommended $(n=4,131), 50.9 \%$ received the test compared with $18.5 \%$ of women in the guidelinediscordant group $(\mathrm{n}=1,163)(P<.001)$.

Women who received the ODx test were significantly younger than those who did not (mean age, 58 vs 66 years; $P<.001$ ). Married women and women residing in affluent neighborhoods had higher rates of ODx testing $(P<.001$; Table 1$)$. In our study population, there was a high correlation between race and poverty, with $58 \%$ of white women living in census tracts with $<5 \%$ of families below the poverty line compared with $14 \%$ of black and $32 \%$ of Hispanic women (data not shown). Having health insurance at the time of breast cancer diagnosis was not associated with ODx test receipt in this population.

Overall, a greater proportion of white women received the ODx test compared with nonwhite women (Table 1). There was significant variation in ODx receipt by race and ethnicity in the guideline-concordant and guideline-discordant groups. Among white women for whom the test was recommended, $51.4 \%$ received the test compared with $44.6 \%$ of black women and $47.7 \%$ of Hispanic women (Figure 2). In unadjusted analysis, black women had significantly lower odds of receiving ODx testing (odds ratio [OR], 0.76; 95\% CI, 0.58-1.00) compared with their white counterparts in the guideline-concordant group, whereas Hispanic women (OR, 0.86; 95\% CI, 0.67-1.10) were as likely to receive ODx testing as white women. Older age ( $\geq 65$ years), tumor size $\geq 10 \mathrm{~mm}$, and higher tumor grade were also associated with a lower likelihood of receiving the ODx test in the guideline-concordant group (Table 2). In adjusted logistic regression models, after controlling for age, marital status, and tumor characteristics, nonwhite race was associated with lower odds of receiving ODx testing. Specifically, non-Hispanic black (OR, 0.67; 95\% CI, 0.49-0.92) and Hispanic women (OR, 0.60; 95\% CI, 0.46-0.78) had significantly lower odds of receiving ODx testing (Table 2).

In the guideline-discordant group, $21.2 \%$ of white women received the test compared with $9.0 \%$ of black and $9.7 \%$ of Hispanic women (Figure 2). Both black (OR, 0.37; 95\% CI, 0.17-0.92) and Hispanic women (OR, 0.40; 95\% CI, 0.21-0.76) had significantly de- 
Davis et al

\section{Table 1. Patient Characteristics}

\begin{tabular}{|c|c|c|c|c|}
\hline & & & Received ODx Test & $P$ Value \\
\hline Age, y & & & & $<.001$ \\
\hline Mean (SD) & $63.02(13.66)$ & & $58.60(10.34)$ & \\
\hline \multirow[t]{3}{*}{ Range } & 21-106 & & 23-91 & \\
\hline & $\mathbf{n}$ & & Received ODx Test (\%) & \\
\hline & $(\mathrm{N}=5,294)$ & $\%$ & (Total=37.6\%) & \\
\hline \multicolumn{5}{|l|}{ Demographic characteristics } \\
\hline Age, y & & & & $<.001$ \\
\hline$<50$ & 950 & $17.9 \%$ & $52.4 \%$ & \\
\hline $50-64$ & 1,950 & $36.8 \%$ & $56.9 \%$ & \\
\hline $65-74$ & 1,217 & $23.0 \%$ & $46.7 \%$ & \\
\hline$\geq 75$ & 1,177 & $22.2 \%$ & $12.1 \%$ & \\
\hline Race/Ethnicity ${ }^{a}$ & & & & $<.001$ \\
\hline White & 4,437 & $83.8 \%$ & $45.3 \%$ & \\
\hline Black & 331 & $6.3 \%$ & $32.6 \%$ & \\
\hline Hispanic/Latino & 392 & $7.4 \%$ & $36.7 \%$ & \\
\hline Other & 134 & $2.5 \%$ & $41.8 \%$ & \\
\hline Marital status & & & & $<.001$ \\
\hline Married & 2,885 & $54.5 \%$ & $50.5 \%$ & \\
\hline Not married & 2,279 & $43.0 \%$ & $35.3 \%$ & \\
\hline Unknown & 130 & $2.5 \%$ & $43.1 \%$ & \\
\hline Neighborhood poverty & & & & $<.001$ \\
\hline$<5 \%$ & 2,765 & $52.2 \%$ & $46.7 \%$ & \\
\hline $5 \%$ to $<10 \%$ & 1,332 & $25.2 \%$ & $43.9 \%$ & \\
\hline $10 \%-100 \%$ & 1,184 & $22.4 \%$ & $36.2 \%$ & \\
\hline Unknown & 13 & $0.2 \%$ & $76.9 \%$ & \\
\hline Health insurance at diagnosis & & & & .11 \\
\hline No insurance & 42 & $0.8 \%$ & $28.6 \%$ & \\
\hline Insurance & 5,123 & $96.8 \%$ & $44.0 \%$ & \\
\hline Other/unknown & 129 & $2.4 \%$ & $41.1 \%$ & \\
\hline \multicolumn{5}{|l|}{ Clinical characteristics } \\
\hline NCCN-recommended ODx use & & & & $<.001$ \\
\hline Guideline-concordant & 4,131 & $73.0 \%$ & $50.9 \%$ & \\
\hline Guideline-discordant & 1,163 & $22.0 \%$ & $18.5 \%$ & \\
\hline Tumor grade & & & & $<.001$ \\
\hline Grade I & 1,262 & $23.7 \%$ & $38.2 \%$ & \\
\hline Grade II & 2,676 & $50.2 \%$ & $39.5 \%$ & \\
\hline Grade III & 1,060 & $19.9 \%$ & $23.6 \%$ & \\
\hline Unknown/NA & 332 & $6.2 \%$ & $23.4 \%$ & \\
\hline Tumor size, mm & & & & $<.001$ \\
\hline$<5$ & 16 & $0.3 \%$ & $6.3 \%$ & \\
\hline $5-9$ & 1,030 & $19.5 \%$ & $41.9 \%$ & \\
\hline $10-29$ & 3,250 & $61.4 \%$ & $50.8 \%$ & \\
\hline$\geq 30$ & 970 & $18.3 \%$ & $24.0 \%$ & \\
\hline Unknown & 28 & $0.5 \%$ & $0.0 \%$ & \\
\hline Lymph node status & & & & $<.001$ \\
\hline No nodes & 3,881 & $73.3 \%$ & $51.0 \%$ & \\
\hline Micrometastasis & 250 & $4.7 \%$ & $49.6 \%$ & \\
\hline Macrometastasis & 1,163 & $22.0 \%$ & $18.5 \%$ & \\
\hline
\end{tabular}

Abbreviations: NA, not available; ODx, Oncotype DX.

aRace and ethnicity categorized into Hispanic, non-Hispanic white, non-Hispanic black, and non-Hispanic other. 
Disparities in Oncotype DX Adoption

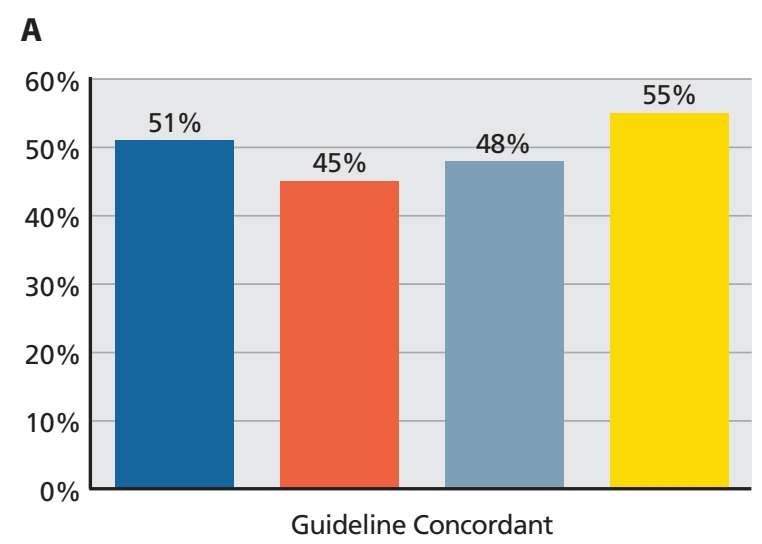

Non-Hispanic white

Non-Hispanic black

\section{B}

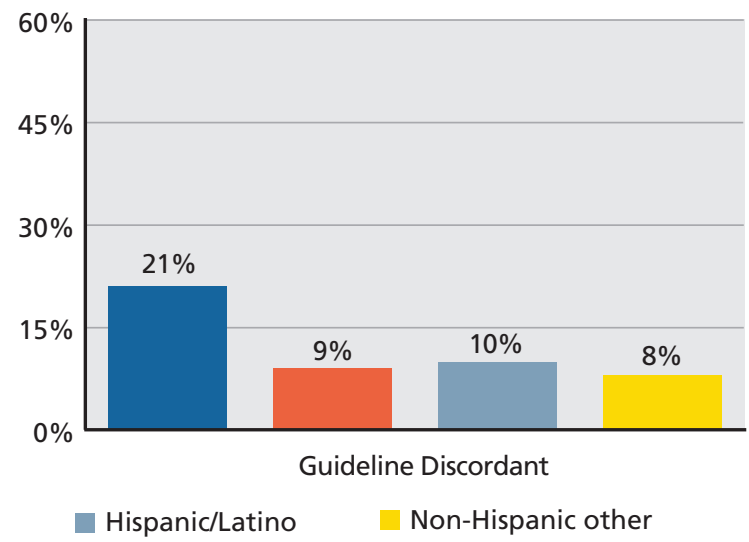

Figure 2. Racial and ethnic variation in Oncotype DX use in Connecticut (2011-2013) by NCCN Guideline (A) concordance and (B) discordance.

creased odds of receiving ODx testing in unadjusted analysis. Similar to the guideline-concordant group, higher tumor grade was associated with a decrease in the likelihood of receiving the ODx test (Table 3). In the adjusted analysis, the likelihood of receiving ODx testing remained low for non-Hispanic black women (OR, 0.42; 95\% CI, 0.21-0.83) and Hispanic women (OR, 0.47; 95\% CI, 0.24-0.91) compared with white women (Table 3). Women in the 65- to 74-year age group were less likely to receive guidelinerecommended ODx testing compared with women aged $<50$ years (OR, 0.43; 95\% CI, 0.34-0.53; Table 2 ), but were more likely to receive the test compared with women for whom the test was not recommended (OR, 1.86; 95\% CI, 1.17-2.95; Table 3).

\section{Discussion}

In this population-based study, we found that race and ethnicity were associated with the likelihood of receiving the ODx test among women with early-stage invasive breast cancer. This was persistent among women for whom NCCN recommends considering the test (guideline-concordant) due to its prognostic and predictive capabilities in improving breast cancer care, as well as among women for whom the test is not recommended (guideline-discordant). Our findings of ODx use rates among guideline-concordant $(51 \%)$ and guideline-discordant $(18 \%)$ populations are similar to those of other studies. ${ }^{17,18}$ However, the persistence of racial disparities in both recommended and nonrecommended groups was not consistent with other examinations of race and ODx receipt. ${ }^{16,23}$
Our study expands on the limited literature on ODx receipt among Hispanic women. We found no difference in guideline-concordant use, but significantly lower discordant use among Hispanic women compared with white women. Additionally, we found that marital status and age were associated with $\mathrm{ODx}$ test receipt in the guideline-concordant group. Previous studies have reported mixed findings on $\mathrm{ODx}$ receipt and marital status. ${ }^{25}$ Our findings corroborate prior reports of decreased use among older women. ${ }^{16,18,21,22}$ Some have posited that the perceived benefit of chemotherapy at baseline may influence the use of the ODx test as a decision-making aid. ${ }^{14}$

Several factors distinguish our study from similar studies on this topic. Our study is statewide, population-based, and not restricted to a given population, as may be the case with other studies. Because ODx testing is more prevalent among younger women, and because black women specifically are more likely to present at a younger age, we likely had more statistical power to detect racial differences in this larger, more diverse population. ${ }^{15}$ As a population-based analysis, our study also circumvents disparities that may result from study enrollment. All women diagnosed with breast cancer in Connecticut had an opportunity to be included in this study, which reduced bias associated with provider type, location, or patient mistrust of academic research studies in our analysis. Our study also had a more robust ascertainment of ODx testing, and in contrast to prior studies on disparities that only assessed race, ${ }^{16,20,23}$ we specifically assessed disparities among women of Hispanic ethnicity. 
Davis et al

\begin{tabular}{|c|c|c|c|c|c|c|c|c|}
\hline \multirow[b]{2}{*}{ Variable } & \multicolumn{4}{|c|}{ Unadjusted Model } & \multicolumn{4}{|c|}{ Adjusted Model } \\
\hline & OR & & & $P$ Value & OR & & & $P$ Value \\
\hline \multicolumn{9}{|l|}{ Age, y } \\
\hline$<50$ & 1.00 & & & & 1.00 & & & \\
\hline $50-64$ & 0.89 & 0.73 & 1.09 & .25 & 0.89 & 0.72 & 1.09 & .25 \\
\hline $65-74$ & 0.43 & 0.35 & 0.53 & $<.001$ & 0.43 & 0.34 & 0.53 & $<.001$ \\
\hline$\geq 75$ & 0.06 & 0.05 & 0.07 & $<.001$ & 0.06 & 0.04 & 0.07 & $<.001$ \\
\hline \multicolumn{9}{|l|}{ Race/Ethnicitya } \\
\hline White & 1.00 & & & & 1.00 & & & \\
\hline Black & 0.76 & 0.58 & 1.00 & .05 & 0.67 & 0.49 & 0.92 & .01 \\
\hline Hispanic & 0.86 & 0.67 & 1.10 & .23 & 0.60 & 0.46 & 0.78 & $<.001$ \\
\hline Other & 1.14 & 0.76 & 1.71 & .53 & 0.65 & 0.42 & 1.01 & .06 \\
\hline \multicolumn{9}{|l|}{ Marital status } \\
\hline Married & 1.00 & & & & 1.00 & & & \\
\hline Not married & 0.5 & 0.44 & 0.57 & $<.001$ & 0.80 & 0.69 & 0.93 & $<.01$ \\
\hline Unknown & 0.82 & 0.55 & 1.23 & .35 & 0.99 & 0.62 & 1.59 & .98 \\
\hline \multicolumn{9}{|l|}{ Tumor size, mm } \\
\hline $5-9$ & 1.00 & & & & 1.00 & & & \\
\hline $10-29$ & 1.82 & 1.57 & 2.11 & $<.001$ & 2.20 & 1.85 & 2.60 & $<.001$ \\
\hline$\geq 30$ & 0.77 & 61.0 & 0.96 & .02 & 0.94 & 0.72 & 1.21 & .62 \\
\hline \multicolumn{9}{|l|}{ Tumor grade } \\
\hline 1 & 1.00 & & & & 1.00 & & & \\
\hline 2 & 1.29 & 1.12 & 1.49 & .001 & 1.27 & 1.08 & 1.51 & $<.01$ \\
\hline 3 & 1.21 & 1.00 & 1.47 & .05 & 1.01 & 0.81 & 1.27 & .93 \\
\hline Unknown & 0.79 & 0.60 & 1.04 & .09 & 0.81 & 0.59 & 1.11 & .18 \\
\hline
\end{tabular}

Abbreviation: OR, odds ratio.

aRace and ethnicity categorized into Hispanic, non-Hispanic white, non-Hispanic black, and non-Hispanic other.

Additionally, our study acknowledges the confounding relationship between race and socioeconomic status, particularly in the adoption of medical technology. Several studies that did not observe racial disparities in ODx adoption included either family income, residential poverty, or the place at which care was received in their models, which may attenuate the relationship between race and ODx adoption, despite the high correlation between race and lower socioeconomic status in the United States. ${ }^{24,25}$ In our study, nonwhite women were significantly more likely to live in high-poverty areas, and in exploratory analyses, poverty did not meaningfully change the effect size in our model. As such, we concluded that the inclusion of poverty in our model would overcontrol and thus artificially mask the true relationship between race, ethnicity, and access to ODx. ${ }^{28}$

Our study also had limitations. We did not distinguish between type of facility where care was received, which may impact likelihood of being offered the test. ${ }^{20}$ However, we believe its exclusion does not reduce our ability to identify true disparities in ODx uptake. Our study was also unable to distinguish between women who declined the test and those for whom the test was never offered. Likewise, our study did not explore willingness of different populations to undergo chemotherapy, which could also impact use of this test, because it is only logical to pursue testing if the patient will undergo the test-advised therapy. Mistrust could possibly impact ultimate use of ODx in nonwhite populations; however, studies suggest that among women who did not receive the test, most were not offered the test and had not heard of it. ${ }^{22}$ Finally, our study focused only on one state, which could potentially reduce generalizability, despite its population-based methodology. However, it can serve as an important step to understanding socioeconomic, racial, and ethnic disparities.

The intended role of clinical guidelines is to remove subjectivity from decision-making, likely reducing disparities in access and treatment. The 
Disparities in Oncotype DX Adoption

\begin{tabular}{|c|c|c|c|c|c|c|c|c|}
\hline \multirow[b]{2}{*}{ Variable } & \multicolumn{4}{|c|}{ Unadjusted Model } & \multicolumn{4}{|c|}{ Adjusted Model } \\
\hline & OR & & & $P$ Value & OR & & & $P$ Value \\
\hline \multicolumn{9}{|l|}{ Age, y } \\
\hline$<50$ & 1.00 & & & & 1.00 & & & \\
\hline $50-64$ & 1.47 & 0.99 & 2.18 & .06 & 1.35 & 0.89 & 2.04 & .16 \\
\hline $65-74$ & 2.25 & 0.45 & 3.49 & $<.001$ & 1.86 & 1.17 & 2.95 & .01 \\
\hline$\geq 75$ & 0.81 & 0.47 & 1.39 & .45 & 0.70 & 0.39 & 1.24 & .22 \\
\hline \multicolumn{9}{|l|}{ Race/Ethnicity ${ }^{\mathrm{a}}$} \\
\hline White & 1.00 & & & & 1.00 & & & \\
\hline Black & 0.37 & 0.19 & 0.72 & .004 & 0.42 & 0.21 & 0.83 & .01 \\
\hline Hispanic & 0.40 & 0.21 & 0.76 & .01 & 0.47 & 0.24 & 0.91 & .02 \\
\hline Other & 0.33 & 0.10 & 1.08 & .07 & 0.40 & 0.12 & 1.33 & .13 \\
\hline \multicolumn{9}{|l|}{ Marital status } \\
\hline Married & 1.00 & & & & 1.00 & & & \\
\hline Not married & 0.66 & 0.49 & 0.9 & .01 & 0.76 & 0.54 & 1.06 & .1 \\
\hline Unknown & 0.82 & 0.12 & 1.3 & .13 & 0.39 & 0.11 & 1.34 & .13 \\
\hline \multicolumn{9}{|l|}{ Tumor size, $\mathrm{mm}$} \\
\hline$<5$ & 1.00 & & & & 1.00 & & & \\
\hline $5-9$ & 9.31 & 1.13 & 76.64 & .04 & 8.83 & 1.03 & 75.44 & .05 \\
\hline $10-29$ & 4.48 & 1.57 & 34.25 & .15 & 4.20 & 0.54 & 32.95 & .17 \\
\hline$\geq 30$ & 2.11 & 61.0 & 16.28 & .47 & 2.12 & 0.27 & 16.8 & .48 \\
\hline \multicolumn{9}{|l|}{ Tumor grade } \\
\hline 1 & 1.00 & & & & 1.00 & & & \\
\hline 2 & 0.60 & 0.39 & 0.92 & .02 & 0.75 & 0.47 & 1.17 & .2 \\
\hline 3 & 0.24 & 0.15 & 0.40 & $<.001$ & 0.33 & 0.19 & 0.56 & $<.001$ \\
\hline Unknown & 0.34 & 0.16 & 0.71 & .004 & 0.52 & 0.24 & 1.13 & .1 \\
\hline
\end{tabular}

Abbreviation: OR, odds ratio.

aRace and ethnicity categorized into Hispanic, non-Hispanic white, non-Hispanic black, and non-Hispanic other.

disparities in uptake found in this and similar studies suggest that nonclinical factors are driving use. Moreover, early diffusion of medical advancements to new target groups may widen racial and ethnic gaps through disparate access to innovation. It is critical that those who currently determine access to resources are intentional about equity in care. Clinicians, policy-makers, and other stakeholders should aim to understand and act on disparities in access for not only cancer treatment but also important risk stratification approaches.

\section{Conclusions}

Observed racial and ethnic disparities in ODx testing are particularly concerning given its potential to guide early-stage, invasive, $\mathrm{HR}+$ breast cancer care, and further exacerbate disparities in treatment quality, survival, and quality of life. This study extends the knowledge base on ODx adoption at a population level and highlights guideline-discordant use. Future studies examining the cause of racial and ethnic disparities as well as mitigation strategies are imperative to narrow disparities in breast cancer outcomes.

\section{References}

1. Bigby J, Holmes M. Disparities across the breast cancer continuum. Cancer Causes Control 2005;16:35-44.

2. Elmore JG, Nakano CY, Linden HM, et al. Racial inequities in the timing of breast cancer detection, diagnosis, and initiation of treatment. Med Care 2005;43:141-148.

3. Fedewa SA, Ward EM, Stewart AK, Edge SB. Delays in adjuvant chemotherapy treatment among patients with breast cancer are more likely in African American and Hispanic ppoulations: a national cohort study 2004-2006. J Clin Oncol 2010;28:4135-4141.

4. Adams SA, Smith ER, Hardin J, et al. Racial differnces in follow-up of abnormal mammography findings among economically disadvantaged women. Cancer 2009;115:5788-5797.

5. Bickell NA, Wang JJ, Oluwole S, et al. Missed opportunities: racial disparities in adjuvant breast cancer treatment. J Clin Oncol 2006;24:13571362 . 
Davis et al

6. McCarthy EP, Burns RB, Coughlin SS, et al. Mammography use helps to explain differences in breast cancer stage at diagnosis between older black and white women. Ann Intern Med 1998;128:729-736.

7. Ward E, Jemal A, Cokkinides V, et al. Cancer disparities by race/ethnicity and socioeconomic status. CA Cancer J Clin 2004;54:78-93.

8. van Ravesteyn NT, Schechter CB, Near AM, et al. Race-specific impact of natural history, mammography screening, and adjuvant treatment on breast cancer mortality rates in the United States. Cancer Epidemiol Biomarkers Prev 2011;20:112-122.

9. Tehranifar P, Neugut AI, Phelan JC, Link BG, et al. Medical advances and racial/ethnic disparities in cancer survival. Cancer Epidemiol Biomarkers Prev 2009;18:2701-2708.

10. Paik S, Tang G, Shak S, et al. Gene expression and benefit of chemotherapy in women with node-negative, estrogen receptor-positive breast cancer. J Clin Oncol 2006;24:3726-3734.

11. Gradishar WJ, Anderson BO, Blair SL, et al. NCCN Clinical Practice Guidelines in Oncology: Breast Cancer, version 3.2014. Accessed September 14, 2014. To view the most recent version of these guidelines, visit NCCN.org.

12. Carlson RW, Allred DC, Anderson BO, et al. Breast cancer. Clinical practice guidelines in oncology. J Natl Compr Canc Netw 2009;7:122-192.

13. Gradishar WJ, Anderson BO, Balassanian R, et al. NCCN Clinical Practice Guidelines in Oncology: Breast Cancer, version 3.2015. Accessed October 10, 2016. To view the most recent version of these guidelines, visit NCCN.org.

14. Su KW, Hall J, Soulos PR, et al. Association of 21-gene recurrence score assay and adjuvant chemotherapy use in the medicare population, 2008. 2011. J Geriatric Oncol 2016;7:15-23.

15. Sheppard VB, O'Neill SC, Dilawari A, et al. Patterns of 21-gene assay testing and chemotherapy use in black and white breast cancer patients. Clin Breast Cancer 2015;15:e83-92.

16. Roberts MC, Weinberger M, Dusetzina SB, et al. Racial variation in the uptake of Oncotype DX testing for early-stage breast cancer. J Clin Oncol 2016;34:130-138.
17. Lund MJ, Mosunjac M, Davis KM, et al. 21-Gene recurrence scores: racial differences in testing, scores, treatment, and outcome. Cancer 2012;118:788-796.

18. Hassett MJ, Silver SM, Hughes ME, et al. Adoption of gene expression profile testing and association with use of chemotherapy among women with breast cancer. J Clin Oncol 2012;30:2218-2226.

19. Haas JS, Phillips KA, Liang SY, et al. Genomic testing and therapies for breast cancer in clinical practice. J Clin Oncol 2011;7(3 Suppl):e1s-7s.

20. Guth AA, Fineberg S, Fei K, et al. Utilization of Oncotype DX in an inner city population: race or place? Int J Breast Cancer 2013;2013:653805.

21. Dinan MA, Mi X, Reed SD, et al. Initial trends in the use of the 21-gene recurrence score assay for patients with breast cancer in the Medicare population, 2005-2009. JAMA Oncol 2015;1:158-166.

22. DeFrank JT, Salz T, Reeder-Hayes K, Brewer NT. Who gets genomic testing for breast cancer recurrence risk? Public Health Genomics 2013;16:215222 .

23. Jasem J, Amini A, Rabinovitch R, et al. 21-gene recurrence score assay as a predictor of adjuvant chemotherapy administration for early-stage breast cancer: an analysis of use, therapeutic implications, and disparity profile. J Clin Oncol 2016;34:1995-2002.

24. Cress RD, Chen YS, Morris CR, et al. Underutilization of gene expression profiling for early-stage breast cancer in California. Cancer Causes Control 2016;27:721-727.

25. O'Neill SC, Isaacs C, Chao C, et al. Adoption of gene expression profiling for breast cancer in US oncology practice for women younger than 65 years. J Natl Compr Canc Netw 2015;13:1216-1224.

26. Gaskin DJ, Dinwiddie GY, Chan KS, McCleary R. Residential segregation and disparities in health care services utilization. Med Care Res Rev 2011;69:158-175.

27. Saloner B, Lê Cook B. Blacks and Hispanics are less likely than whites to complete addiction treatment, largely due to socioeconomic factors. Health Aff (Millwood) 2013;32:135-145.

28. Kawachi I, Daniels N, Robinson DE. Health disparities by race and class: why both matter. Health Aff (Millwood) 2005;24:343-352. 